Laura Arnold* und Ute Teichert

\title{
Politischer Reformprozess im Zuge der \\ COVID-19-Pandemie: Der Pakt für den \\ Öffentlichen Gesundheitsdienst
}

\section{Political reform process in the course of the} COVID 19 pandemic: the pact for public health

\section{services}

https://doi.org/10.1515/pubhef-2020-0130

Zusammenfassung: Im Zuge eines Konjunkturpaketes haben sich Bund und Länder auf einen 4 Milliarden Euro schweren Pakt für den Öffentlichen Gesundheitsdienst (ÖGD) verständigt, um den ÖGD personell wie infrastrukturell zu stärken. Eine zentrale Rolle spielt die nachhaltige Stärkung des ÖGD zur Sicherstellung gesundheitlicher Schutzaufgaben zur Krisenbewältigung. Damit der ÖGD die ihm obliegende komplexe Aufgabe der gesundheitlichen Daseinsfürsorge vor Ort langfristig und qualitativ hochwertig ausfüllen kann, wird es notwendig sein, bestehende Strukturen zu optimieren, so dass akute Bedrohungssituationen ebenso wie eher langfristig und strategisch ausgerichtete Planungsaufgaben souverän gemeistert werden können.

Schlüsselwörter: Öffentlicher Gesundheitsdienst; Organisationsstrukturen im ÖGD; Pakt für den Öffentlichen Gesundheitsdienst; Planung und Steuerung; Public Health Workforce.

\begin{abstract}
The German federal and state governments have agreed on a 4 billion euro pact for the Public Health Services (ÖGD) to strengthen the ÖGD in terms of personnel and infrastructure. The sustainable strengthening of the ÖGD plays a central role to ensure health protection measures for crisis management. In order for the ÖGD to be able to fulfil its complex task of providing high-quality health care on site in the long term, it will be necessary to optimize existing structures so that acute threat situations
\end{abstract}

*Korrespondenz: Laura Arnold, MSc, MA, Akademie für Öffentliches Gesundheitswesen in Düsseldorf, Kanzlerstraße 4, 40472 Düsseldorf, Germany, E-mail: arnold@akademie-oegw.de Dr. Ute Teichert, MPH: Akademie für Öffentliches Gesundheitswesen in Düsseldorf, Düsseldorf, Germany as well as more long-term and strategically oriented planning tasks can be mastered.

Keywords: governance; organizational structures in the public health service; pact for the public health service; public health service; public health workforce.

Im Zuge der COVID-19-Pandemie ist der Öffentliche Gesundheitsdienst (ÖGD) ins mediale Interesse gerückt. $\mathrm{Zu}$ Beginn der Pandemie von der breiten Öffentlichkeit kaum wahrgenommen, vergeht im Herbst 2020 kaum ein Tag, an dem nicht über die Bedeutung des ÖGD mit seinen verschiedenen Institutionen auf kommunaler, Landesund Bundesebene berichtet wird.

Insbesondere den kommunalen Gesundheitsämtern kommt bei der Bewältigung der Pandemie eine zentrale Rolle zu: Nach den Regelungen des Infektionsschutzgesetzes obliegt ihnen die Aufklärung und Unterbrechung der SARS-Cov-2-Infektionsketten. Sie managen u.a. Erkrankungs- und Verdachtsfälle, ermitteln Kontaktpersonen, ordnen Untersuchungen auf eine Infektion an oder führen diese selbst durch und veranlassen im Ernstfall Isolierungs- und Quarantänemaßnahmen [1]. Parallel zur Koordination des Pandemie-Managements vor Ort arbeiten die Gesundheitsämter in kommunalen Krisenstäben mit, koordinieren Sondereinsätze, unterstützen bei der Entwicklung von Hygienekonzepten, beraten Bürgerinnen und Bürger und fungieren als Ansprechpartner für Politik und Fachöffentlichkeit hinsichtlich antiepidemischer Maßnahmen. Das setzt ein engmaschiges Begleitmonitoring voraus, um auf etwaige Änderungen schnellstmöglich reagieren $\mathrm{zu}$ können. Hierzu ist der zeitnahe Transfer wissenschaftlicher Erkenntnisse in den ÖGD hinein und aus dem ÖGD hinaus zwingend notwendig. Da in der aktuellen Situation kaum Zeit dafür ist und es wenig etablierte gemeinsame Forschungsnetzwerke von ÖGD-Praxis und Public Health-Wissenschaft 
gibt, sind die Gesundheitsämter davon abhängig, dass neue Erkenntnisse von Landes- und Bundesbehörden in den ÖGD kommuniziert werden. Aufgrund der zwangsläufig marginalen „Innenperspektive“, fehlt es diesen Lösungsansätzen jedoch oftmals an entsprechender Praxistauglichkeit. Für die dringend notwendige Einbindung der ÖGD-Praxis in die Public Health-Forschung haben die Gesundheitsämter momentan aber keine Zeit.

Allein das Nachverfolgen von Kontakten, was nach Pandemieplan wesentlicher Bestandteil für eine frühe Erkennung und Eindämmung des Ausbruchsgeschehen ist $[2,3]$, hat die ohnehin äußert knappen Personalressourcen des ÖGD nahezu flächendeckend an den Rand des kapazitiv Leistbaren gebracht [4] - von den vielfach liegenbleibenden Pflichtaufgaben einmal ganz zu schweigen. Es war daher nicht verwunderlich, dass sich mit dem Anstieg der Fallzahlen in der medialen Berichterstattung auch zunehmend Berichte über mangelnde Erreichbarkeit der neu eingerichteten Corona-Hotlines oder nicht mehr leistbare Absonderungsmaßnahmen durch den ÖGD fanden. All das trotz der vielerorts oft sehr schnell erfolgten Unterstützung durch zusätzliches Personal durch Medizinstudierende (medis4ÖGD), Containment-Scouts, Personal der Bundeswehr oder im Rahmen von Zuweisungen über Amtshilfe aus anderen Behörden. Politik und Zivilgesellschaft haben zweifelsohne schnell reagiert. Damit der ÖGD seine Aufgaben aber langfristig und nachhaltig qualitativ hochwertig bewältigen kann, bedarf es mehr als einer temporären Unterstützung, so wichtig und dringend notwendig sie während der COVID-19-Pandemie auch war und ist.

Dabei hat die Corona-Krise nicht nur die lange bekannte desolate Personalsituation im ÖGD offengelegt, sondern auch erhebliche strukturelle wie technische Defizite in den Gesundheitsämtern verdeutlicht [5]. Es ist daher nicht verwunderlich, dass die Bundesregierung den ÖGD 2020 gleich mehrfach auf die politische Reformagenda gesetzt hat: Zum einen im Rahmen des zweiten Bevölkerungsschutzgesetzes [6], wonach 50 Millionen Euro bereitgestellt werden, um die Digitalisierung in den Gesundheitsämtern voranzutreiben. Zum anderen im Rahmen des Paktes für den öffentlichen Gesundheitsdienstes [7], wonach sich Bund und Länder auf weitere 4 Milliarden Euro für den Personalaufbau, die Steigerung der Attraktivität des ÖGD, den Weiterausbau der Digitalisierung sowie die Förderung zukunftsfähiger Strukturen innerhalb des ÖGD verständigt haben (siehe auch Tabelle 1).

Die mit dem ÖGD-Pakt einhergehende politische Anerkennung stellt eine Chance dar, den lange Zeit vernachlässigten ÖGD substantiell zu stärken und damit die Bevölkerungsgesundheit in Deutschland unabhängig von zukünftigen Krisen zu schützen und zu fördern [8, 9]. Ob das gelingt, ist jedoch nicht alleine von der Ausgestaltung und Umsetzung des Paktes abhängig, sondern auch von der Veränderungsbereitschaft innerhalb des ÖGD.

Die Corona-Krise hat eindrücklich gezeigt, dass gerade der kommunale ÖGD die Instanz ist, wenn es darum geht Hotspots rechtzeitig $\mathrm{zu}$ identifizieren und Infektionsketten frühestmöglich $\mathrm{zu}$ unterbrechen. Dies ist insofern nicht verwunderlich, als dass die unteren Gesundheitsbehörden aufgrund ihrer kommunalpolitischen Verankerung und dem damit einhergehenden direkten Zugang zu verschiedenen Lebenswelten beste Voraussetzungen haben, das Leitprinzip von „Health in All Policies (HiAP)“ in die Gremien hineinzutragen [10]. Diese strukturellen Rahmenbedingungen spielen auch für Aufgaben außerhalb des klassischen Gesundheitsschutzes, beispielsweise bei der Umsetzung des Präventionsgesetzes [11], eine zentrale Rolle. Um den ÖGD langfristig zu der im Leitbild ÖGD vorgesehenen zukunfts- und europafähigen Public Health-Agentur weiterzuentwickeln [12, 13], gilt es den ÖGD in ebendiesen Strukturen zu stärken.

Als „dritte Säule“ des Gesundheitswesens übernimmt der ÖGD nicht nur hoheitliche Schutz- und Überwachungsaufgaben, sondern ist gleichermaßen beratend, informierend und begutachtend wie präventiv und gesundheitsförderlich tätig (z.B. im Rahmen des Begutachtungswesens oder der Kinder- und Jugendgesundheit) und verantwortet umfangreiche koordinierende, moderierende sowie politikberatende Tätigkeiten (z.B. im Rahmen der Gesundheitsberichterstattung und -planung) [14]. Hierzu sind wissenschaftliche wie koordinierende Kompetenzen in Steuerungsaufgaben ebenso essentiell wie die Sicherstellung eines ausreichenden und v.a. entsprechend qualifizierten Personals [15]. Um die zentrale Aufgabe der gesundheitlichen Daseinsfürsorge souverän meistern zu können, wird der ÖGD zukünftig auch in der Lage sein müssen, zwischen akuten Bedrohungssituationen im Sinne einer kurzfristigen Gefahrenabwehr und den eher langfristig und strategisch ausgerichteten Planungsaufgaben zur Sicherstellung gesundheitlicher Chancengleichheit wechseln zu können. Dies setzt umfangreiche Kompetenzen in den beiden Bereichen voraus und erfordert ressortinterne wie -übergreifende Kooperationen auf Augenhöhe.

Um eine solche komplexe Aufgaben- und Verwaltungsstruktur erfolgreich implementieren zu können, gilt es zusätzlich zu den im ÖGD-Pakt vorgesehenen Maßnahmen, auch die ÖGD-internen Strukturen kritisch zu prüfen und bestehende Hindernisse abzubauen. Der (kommunale) ÖGD ist bei entsprechender Ausstattung wie kein anderer Akteur in der Lage, bei der Erarbeitung evidenzbasierter Lösungsansätze zu unterstützen und 
Tabelle 1: Im Pakt für den Öffentlichen Gesundheitsdienst vorgesehenen Maßnahmen im Auszug [7].

\begin{tabular}{|c|c|}
\hline Personalaufbau & $\begin{array}{l}\text { - Schaffung neuer Stellen (min } 1.500 \text { neue, unbefristete VZÄ bis Ende 2021; min. weitere } 3.500 \text { VZÄ bis } \\
\text { Ende 2022) } \\
\text { - Vorlage konkreter Personalaufwuchskonzepte und -zielsetzungen bis Ende 2021, wobei } 90 \% \text { des } \\
\text { Personalaufbaus in den unteren Gesundheitsbehörden/den örtlichen Gesundheitsämtern erfolgen soll; } \\
\text { Die personelle Verbesserung soll sich an der Aufgabenbeschreibung im Leitbild ÖGD orientieren } \\
\text { - Schaffung } 40 \text { neuer Stellen in der Bundesverwaltung zur Maßnahmenumsetzung und Evaluierung }\end{array}$ \\
\hline Digitalisierung & $\begin{array}{l}\text { - DEMIS soll unter Berücksichtigung bestehender Systeme (wie z.B. SORMAS) als gemeinsame } \\
\text { Kommunikationsplattform bis Ende } 2022 \text { allen Gesundheitsbehörden zur Verfügung stehen } \\
\text { - Bund stellt } 50 \text { Millionen Euro zur technischen Modernisierung des ÖGD zur Verfügung } \\
\text { - Vereinbarung zentraler Standards zur Sicherstellung einer übergreifenden Kommunikation und } \\
\text { Interoperabilität } \\
\text { - Länder verpflichten sich die gemeinsam bis zum Frühjahr unter Einbezug relevanter Akteure } \\
\text { abgestimmten Mindeststandards einzuhalten („Digitales Gesundheitsamt } 2025 “ \text { ); Implementation und } \\
\text { Evaluation erfolgt im Rahmen eines begleitenden Forschungsvorhaben des BMG }\end{array}$ \\
\hline $\begin{array}{l}\text { Steigerung der } \\
\text { Attraktivität des ÖGD }\end{array}$ & $\begin{array}{l}\text { - Länder streben unter Beachtung der Tarifautonomie eine attraktive Bezahlung für das ärztliche Personal } \\
\text { im ÖGD sowie eine Verbesserung für das beamtete ärztliche Personal im ÖGD an } \\
\text { - Vertiefung der Verbindung des ÖGD mit der Wissenschaft sowohl in der Fort-, Aus- und Weiterbildung als } \\
\text { auch in der Forschung } \\
\text { - Öffentliches Gesundheitswesen und Bevölkerungsmedizin sollen thematisch stärker in der } \\
\text { medizinischen Ausbildung verankert werden } \\
\text { - Länder bitten die Ärztekammern zu prüfen, inwiefern extern im ÖGW erworbene Kenntnisse, Erfahrungen } \\
\text { und Fertigkeiten auf die Weiterbildung angerechnet werden können } \\
\text { - Förderung von Forschungsprogrammen zur Stärkung der wissenschaftlichen Grundlage des ÖGD und } \\
\text { Prüfung der Fördermöglichkeit struktureller Maßnahmen zum Ausbau von Lehre und Forschung auf dem } \\
\text { Gebiet des ÖGW } \\
\text { - Stärkung der Fort-, Aus- und Weiterbildung für die Fachberufe im ÖGD, um dem Mangel an } \\
\text { (medizinischem) Fachpersonal entgegenzuwirken } \\
\text { - Kommunikation zur Tätigkeit und Bedeutung des ÖGD auf allen Ebenen verbessern und ausbauen } \\
\text { inkl. gemeinsamer Kampagne zur stärkeren Sichtbarmachung der Aufgabenbreite des ÖGD und seiner } \\
\text { Bedeutung für den Gesundheitsschutz der Bevölkerung }\end{array}$ \\
\hline Umsetzung der IGV & $\begin{array}{l}\text { - Stärkung notwendiger Strukturen zur Umsetzung der Internationalen Gesundheitsvorschriften (IGV) im } \\
\text { Rahmen eines Förderprogrammes in Höhe von } 50 \text { Millionen Euro }\end{array}$ \\
\hline Zukunftsfähige & - Bessere strukturelle Aufstellung des ÖGD auf akute Herausforderungen \\
\hline Strukturen des ÖGD & $\begin{array}{l}\text { - Einberufung eines externen und unabhängigen Expertenbeirats, um den ÖGD für kommende Pandemien } \\
\text { und andere nationale gesundheitliche Notlagen organisatorisch und rechtlich vorzubereiten; Bis Ende } \\
2021 \text { definieren Bund und Länder weitere strukturelle Anpassungen und legen einen Umsetzungsplan } \\
\text { vor, der das von der GMK verabschiedete Leitbild berücksichtig. } \\
\text { - Grundlage für die Planung von Maßnahmen sowohl in Bezug auf Gesundheitsförderung, Prävention, } \\
\text { Beratung als auch Krisenmanagement bildet eine gute Gesundheitsberichterstattung }\end{array}$ \\
\hline
\end{tabular}

Abkürzungen: BMG - Bundesministerium für Gesundheit; ÖGD - Öffentlicher Gesundheitsdienst; ÖGW - Öffentliches Gesundheitswesen; VZÄ - Vollzeitäquivalent; IGV - Internationale Gesundheitsvorschriften.

passgenaue Maßnahmen zu entwickeln. Gleichzeitig kann er in vermittelnder sowie eigens aktiver Funktion auch bei der konkreten Umsetzung und Evaluation eine zentrale Rolle übernehmen. All das unabhängig davon, ob es sich hierbei um kurzfristig zur Krisenbewältigung oder langfristig zur Daseinsfürsorge ausgerichtete Maßnahmen handelt. Ein solches „intelligentes Switchen“ zwischen den verschiedenen Bereichen setzt ein beachtliches Maß wissenschaftlicher Kompetenz voraus, was neben integrierten Ausbildungskonzepten von Public HealthWissenschaft und ÖGD-Praxis auch ein klares Bekenntnis vonseiten des ÖGD zur Stärkung der ÖGD-Wissenschaft und ÖGD-relevanten Forschung in Deutschland benötigt [16]. Das Ziel muss sein, von einem weitestgehend „beforschten“ Akteur zum „forschenden“ Akteur zu werden, der sich die Implementierung von HiAP auf die Fahnen schreibt, und dabei sowohl das traditionelle Aufgabengebiet des Gesundheitsschutzes sowie den (kommunalen) Planungs- und Steuerungsbereich im Blick behält.

\section{Autorenerklärung}

Autorenbeteiligung: Alle Autorinnen tragen Verantwortung für den gesamten Inhalt dieses Artikels und haben der Einreichung des Manuskripts zugestimmt. Finanzierung: 
Die Autorinnen erklären, dass sie keine finanzielle Förderung erhalten haben. Interessenkonflikt: Die Autorinnen erklären, dass kein wirtschaftlicher oder persönlicher Interessenkonflikt vorliegt. LA ist als Referentin für Epidemiologie und Gesundheitsberichterstattung an der Akademie für Öffentliches Gesundheitswesen in Düsseldorf tätig und Sprecherin des Nachwuchsnetzwerkes Öffentliche Gesundheit (NÖG). UT ist Leiterin der Akademie für Öffentliches Gesundheitswesen in Düsseldorf und Vorsitzende des Bundesverbandes für Ärztinnen und Ärzte im Öffentlichen Gesundheitsdienst e.V. (BVÖGD).

\section{Author Declaration}

Author contributions: All authors have accepted responsibility for the entire content of this submitted manuscript and approved submission. Funding: Authors state no funding involved. Conflict of interest: Authors state no conflict of interest. LA works as a consultant for epidemiology and health reporting at the Academy for Public Health Services in Düsseldorf and spokesperson of the German Network of Young Professionals in Public Health. UT is director of the Academy for Public Health Services in Düsseldorf and chairwoman of the Federal Association of Physicians of the Public Health Service.

\section{Literatur}

1. Starke D. Arbeitsalltag in der Krise. Die Rolle der Gesundheitsämter in der Corona-Pandemie. Dr. med. Mabuse 2020:245.

2. RKI. Ergänzung zum Nationalen Pandemieplan - COVID-19 neuartige Coronaviruserkrankung. In: Robert Koch-Institut, Hg. Berlin, 2020, 04.03.2020.

3. RKI. Nationaler Pandemieplan Teil II - Wissenschaftliche Grundlagen. In: Robert Koch-Institut, Hg. Berlin, 2016.

4. Mast M, Gutensohn D, Erdmann E, Blickle P, Fischer L, Schöps C, et al. Gesundheitsämter: Hat Deutschland Corona unter Kontrolle? Die Zeit. 19.06.2020, 2020. Online verfügbar unter https:// www.zeit.de/wissen/gesundheit/2020-06/gesundheitsaemtercorona-infektionsketten-nachverfolgung-meldeverfahren-tests/ komplettansicht, zuletzt geprüft am 30.11.2020.

5. Gruhl M. Quo vadis, ÖGD? In: Observer Gesundheit, Hg., 08.07.2020, 2020. Online verfügbar unter https://observergesundheit.de/quo-vadis-oegd/, zuletzt geprüft am 29.11.2020.
6. Bundestag. Zweites Gesetz zum Schutz der Bevölkerung bei einer epidemischen Lage von nationaler Tragweite. Bundesgesundheitsbl, 19.05.2020, 2020.

7. Bundesgesundheitsministerium. Pakt für den öffentlichen Gesundheitsdienst, 22.10.2020, 2020. Online verfügbar unter https://www.bundesgesundheitsministerium.de/service/begriffe-von-a-z/o/oeffentlicher-gesundheitsheitsdienst-pakt.html, zuletzt geprüft am 22.10.2020.

8. Zukunftsforum Public Health. Der Pakt für den Öffentlichen Gesundheitsdienst. Empfehlungen für Umsetzung und Ausgestaltung. Empfehlungen für Umsetzung und Ausgestaltung, 08.07.2020, 2020. Online verfügbar unter https://zukunftsforum-public-health.de/stellungnahme-pakt-oegd/, zuletzt geprüft am 01.09.2020.

9. BVÖGD. 10 Punkte Plan zur Stärkung des ÖGD. In: Bundesverband der Ärztinnen und Ärzte des Öffentlichen Gesundheitsdienstes e.V., Hg., 15.07.2020, 2020. Online verfügbar unter https://www.bvoegd.de/bvoegd-legt-10-punkte-plan-zur-nachhaltigen-staerkung-des-oegd-vor-den-worten-muessen-nuntaten-folgen/, zuletzt geprüft am 04.11.2020.

10. Geene R, Gerhardus A, Grossmann B, Kuhn J, Kurth BM, Moebus S, et al. Health in All Policies - Entwicklungen, Schwerpunkte und Umsetzungsstrategien für Deutschland. Ausgearbeitet in einer AG des Zukunftsforums Public Health in Kooperation mit der Bundesvereinigung Prävention und Gesundheitsförderung und Gesundheit Berlin-Brandenburg. Zukunftsforum Public Health, Hg. 2020. Online verfügbar unter https://zukunftsforum-public-health.de/health-in-all-policies/, zuletzt geprüft am 01.03.2020.

11. Szagun B, Kuhn J, Starke D. Kommunale Gesundheitsförderungspolitik und das Präventionsgesetz. Präv Gesundheitsf 2016;11:265-70.

12. Länderoffene Projektgruppe „Leitbild ÖGD“. Konsens der länderoffenen Arbeitsgruppe zu einem Leitbild für einen modernen Öffentlichen Gesundheitsdienst. Gesundheitswesen 2018;80:679-81.

13. Kuhn J, Wildner M. Corona-Krise und öffentlicher Gesundheitsdienst. GGW 2020;20.

14. KGSt. Ziele, Leistungen und Steuerung des kommunalen Gesundheitsdienstes. KGSt-Bericht 11/1998. Unter Mitarbeit von Rainer Heinz. In: Kommunale Gemeinschaftsstelle, Hg. Köln, 1998 (Az.: 53000 ).

15. Teichert U, Bräunling S, Müller K, Bär G. Praxis von Public Health. Gesundheitswesen 2017;79:940-3.

16. Teichert U, Kaufhold C, Rissland J, Tinnemann P, Wildner M. Vorschlag für ein bundesweites Johann-Peter Frank Kooperationsmodell im Rahmen der nationalen Leopoldina-Initiative für Public Health und Global Health. Gesundheitswesen 2016;78:473-6. 